„DIE ZUNEHMENDEN REGLEMENTIERUNGEN STELLEN DEN

WISSENSSTANDORT DEUTSCHLAND INFRAGE UND REDUZIEREN DIE MÖGLICHKEITEN ZU EINEM WISSENSBASIERTEN DIALOG MIT DER ÖFFENTLICHKEIT.“

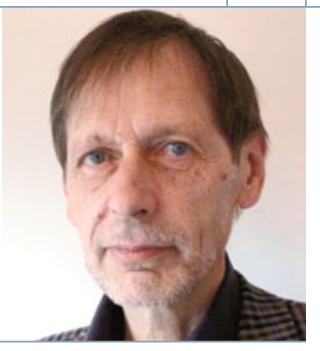

Wolfgang Nellen

\title{
Die BASF geht - Was geht uns das an?
}

Am 16. Januar 2012 gab die BASF bekannt, dass sie die Unternehmensgesellschaft BASF Plant Science in Deutschland einstellt und die Aktivitäten im Bereich „Grüne Gentechnik“ in die USA verlagert. Als Begründung wurde genannt, dass die Akzeptanz in Deutschland nicht vorhanden und Entwicklungsarbeit in Deutschland bzw. Mitteleuropa daher perspektivlos sei. Die Konsequenz: Es werden zukünftig mit gentechnischen Methoden auch keine Pflanzen mehr entwickelt, die an die Bedürfnisse in Mitteleuropa angepasst sind. Ob das wirklich sinnvoll ist, darf bezweifelt werden. Ist die Grüne Gentechnik damit endgültig gescheitert, wie Ulrike Höfgen, Umweltministerin in RheinlandPfalz feststellt?

Fakt ist, dass weltweit ca. 150 Millionen Hektar transgene Nutzpflanzen angebaut werden - Tendenz steigend. Gescheitert ist die Grüne Gentechnik allerdings in Mitteleuropa, wo sie in den 1980er-Jahren mit Jeff Schell und Marc von Montagu begann. Es gelang einer starken, international aufgestellten Interessenvertretung, eine sehr erfolgreiche Protestbewegung zu initiieren, nach und nach ihren Einfluss auszubauen und schließlich sogar Einfluss auf die Sprache zu nehmen („Genmanipulation“, „Genfood“, „Gengemüse“ usw.). Für uns Wissenschaftler ist es erschreckend und deprimierend, dass alle Anstrengungen, die Menschen zu informieren und eine wissensbasierte Diskussion zu führen, gescheitert sind. Die Entscheidung gegen die „Grüne Gentechnik“ fiel fast ausschließlich emotional, solide Forschungsergebnisse spielten dabei so gut wie keine Rolle, wohingegen manch fragwürdige Studie erstaunliche Popularität erlangte.

Der Rückzug der BASF bedeutet nicht, dass in Deutschland die Forschung völlig eingestellt wird, er ist aber ein deutliches Signal und wirft Fragen auf: Inwieweit ist es gerechtfertigt, noch in die relevanten pflanzenbiotechnologischen Studiengänge zu investieren, wenn deren anwendungsorientierte
Absolventen allein für das Ausland ausgebildet werden? Welche Einflussmöglichkeiten hat Deutschland in Zukunft noch in der internationalen Forschung und Entwicklung im Bereich der Grünen Gentechnik?

Last but not least: Was haben wir als Wissenschaftler falsch gemacht? Zunächst nicht viel: Den berühmten „Elfenbeinturm“ gibt es nicht mehr. Forschungsinstitute und Universitäten haben mit viel Engagement die Türen geöffnet, sie haben gute Öffentlichkeitsarbeit geleistet und tun dies noch. Sie beteiligen sich an der Unterrichtsgestaltung in Schulen und stehen zur Diskussion zur Verfügung. Die „Fehler“ liegen meines Erachtens in der Wissenschaft selbst, in ihrer Eigenschaft, Ergebnisse zu hinterfragen und stets Zweifel zu haben. Politiker und Gentechnikgegner machen dagegen klare Aussagen - egal, ob sie nun richtig sind oder nicht. Hinzu kommt, dass ein Vortrag oder eine Laborführung nicht so spektakulär und medienwirksam sind wie ein Traktor-Konvoi oder eine Aktion im Supermarkt (zu der man Wissenschaftler wohl kaum motivieren könnte). Nicht zuletzt hängt der Kampf um die öffentliche Meinung weniger von Argumenten als vom Geld ab. Greenpeace beispielsweise hat in Deutschland ein Spendenaufkommen von knapp 50 Millionen Euro, aus dem rund 160 Mitarbeiter finanziert werden. Von solchen Voraussetzungen können Wissenschaftler nur träumen besonders die stark zersplitterten Biowissenschaften.

Fachgesellschaften übernehmen wichtige Funktionen für ihre Community. Eine noch so fundierte Stellungnahme einer Fachgesellschaft mit 500 oder auch 2.000 Mitgliedern reizt die Medien aber weniger als 50.000 Unterschriften gegen „Gendreck“.

Die einzige Chance, biowissenschaftliche Themen mit gesellschaftlichem Bezug auf einer rationalen Basis in der Öffentlichkeit zu diskutieren, liegt in einer starken, sichtbaren biowissenschaftlichen Community mit all ihren Facetten. Der VBIO arbeitet daran, eine solche Community aufzubauen. Dabei werden wir häufig von Kollegen aus Fachgesellschaften gefragt, was sie denn von einer VBIO-Mitgliedschaft hätten? Einfacher zu beantworten ist die Gegenfrage: Welche Chancen verpassen die Biowissenschaftler ohne eine starke Interessenvertretung? Der Rückzug der BASF Plant Science ist hier nur ein Beispiel von vielen. Änderungen bei Tierschutz, Biostoffverordnung, Gentechnikgesetz, Gendiagnostik, Tiertransporten und anderes mehr werden die Arbeitsmöglichkeiten vieler Biowissenschaftler und die Ausbildung des Nachwuchses an Schule und Hochschule beeinträchtigen. Auf lange Sicht stellen die zunehmenden Reglementierungen damit den Wissensstandort Deutschland infrage und reduzieren die Möglichkeiten, einen wissensbasierten Dialog mit der Öffentlichkeit zu führen, noch weiter.

Daher ist es geradezu „überlebenswichtig“, dass wir Biowissenschaftler jenseits der Fächergrenzen gemeinsam mit einer kräftigen Stimme sprechen. Und diese kräftige Stimme kann nach Lage der Dinge eigentlich nur der VBIO sein, davon bin ich überzeugt.

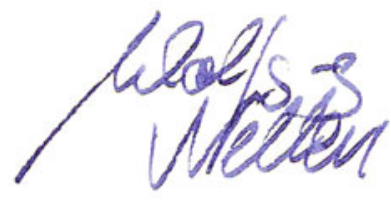

Wolfgang Nellen, Präsident des VBIO

Korrespondenzadresse: Prof. Dr. Wolfgang Nellen Abteilung Genetik Universität Kassel Heinrich-Plett-Straße 40 D-34132 Kassel Tel.: 0561-8044805 Fax: 0561-804-4800 nellen@uni-kassel.de 